\title{
Application of Autologous Cartilage Transplantation in the Repair and Reconstruction of Nasal Soft-Tissue Triangle Deformities
}

\author{
Ma Z, Wang Y, Chen G, Wang J, Zhang J and Huang J* \\ Department of plastic surgery, Hospital of Nanjing University of Chinese medicine, \\ China
}

*Corresponding author: Jinlong Huang, Department of plastic surgery of affiliated Hospital of Nanjing University of Chinese medicine, Nanjing Jiangsu 210000, China, Email: jsmrzx@163.com

\begin{tabular}{|c|}
\hline Research article \\
Volume 3 Special Issue 2 \\
Received Date: July 02, 2019 \\
Published Date: July 15, 2019 \\
DOI: $10.23880 /$ ijtps-16000S2-007 \\
\hline
\end{tabular}

\section{Abstract}

Objective: To evaluate the effect of autologous cartilage transplantation in repairing nasal soft-tissue triangle deformities.

Methods: From January 2015 to January 2018, 23 patients underwent the surgery, got part of the costal cartilage, nasal septum cartilage or ear cartilage, An open incision was made along the medial nasal columella and lateral rim of nostril, the cartilage was carved into strips as a graft of the lateral and middle crus of the alar cartilage in order to reconstruct the nasal alar dome and correct the nasal soft-tissue triangle deformities. The costal cartilage or nasal septum cartilage was used as columellar strut. At the same time, a prosthetic or autologous costal cartilage scaffold was placed onto the nasal dorsum, and fascia tissue was placed on the tip of the nose to relieve tensions. The changes of the angle of the medial and lateral crus of the alar cartilage before and after autologous cartilage transplantation and the difference between the greatest distance from the long axis of the nostrils to the alar rim before and after the operation were statistically analyzed. The changes of nasal appearance pre and postoperation were compared. To evaluate the correction effect of nasal soft-tissue triangle deformities, bilateral asymmetry and overall surgical effect. The surgical results were evaluated by comparing the pre- and postoperative images, statistical analysis the difference of alar cartilage angle along inside and outside feet before and after the fornix reconstruction, and evaluate the efficacy satisfaction of repairation for retraction, notching, asymmetries of nasal soft-tissue triangle deformities and overall effect of the rhinoplasty.

Results: The alar cartilage angle of the 23 patients was $(50.6 \pm 12.4)^{\circ}$ preoperative, decreased to $(35.0 \pm 5.6)^{\circ}$ when the nasal dome reconstruction was finished, the difference was statistically significant $(P<0.01)$. The patients were followed up for 6 to 18 months, the noses were with natural appearance and tactile impression, and there were no serious complications. The degree of satisfaction was $87.5 \%$. 


\section{International Journal of Transplantation \& Plastic Surgery}

Conclusion: Using autologous cartilage to repair the soft-tissue triangle deformities is an ideal surgical method, the nasal morphology can be improved delicately, and the degree of satisfaction is high postoperatively.

Keywords: Nasal soft-tissue triangle deformities; Autologous cartilage; Reconstruction; Rhinoplasty

\section{Introduction}

Nasal soft-tissue triangle is the region between the lower margin of the dome and the cutaneous margin of the nostrils (Figure 1a). It is the smallest nasal aesthetic subunits, which is the most likely to be neglected in rhinoplasty [1]. A nasal soft-tissue triangle deformity is caused by alar cartilage retraction and its weak support. The main clinical manifestations are alar dome collapse, alar margin retraction and bilateral soft-tissue triangle asymmetry $[2,3]$. The anatomic feature is that this area is composed only of skin and soft tissue and lacks cartilage support [4-6]. By cartilage transplantation, alar cartilage retraction and collapse can be improved, which can then correct soft-tissue triangle deformities [1-3,5]. Thereby, clinical success can be achieved from aesthetic and functional perspectives [7]. Between January 2015 and January 2018, this method was used in 32 patients with soft-tissue triangle deformities. The nasal morphology recovered well after surgery and patients were satisfied. The report is as follows.

\section{Materials and Methods}

\section{Clinical Data}

\section{Inclusion Criteria:}

1) Patients with soft -tissue triangle collapse, and/or alar rim retraction, bilateral soft-tissue triangle asymmetry, etc. combined with other nasal deformities such as flat nose, short nose, wide nose, etc., and received corrective surgery for nasal soft-tissue triangle deformities.

2) The patient was born with soft-tissue triangle deformity and had no history of nasal trauma or surgery.

3) Exclusion of contraindications: hypertension, diabetes, heart disease, pregnancy, etc.

From January 2015 to January 2018, a total of 32 patients with nasal soft-tissue triangle deformities were included in this study, including 24 patients with nasal soft-tissue triangle collapse, 28 patients with alar rim retraction, 3 patients with bilateral incomplete asymmetry, 30 patients with collapsed nose, and 18 patients with short nose. 6 cases were repaired by costal cartilage alone, 11 cases by costal cartilage + prosthesis, 15 cases by nasal septal cartilage + ear cartilage + prosthesis. There were 7 male patients and 25 female patients, aged from 18 to 42 years old, with an average age of $28.5 \pm 6.8$ years old. The patients were followed up for 6 to 18 months, with an average follow-up time of $11.8 \pm 4.1$ months. All patients had informed consent, and this study was approved by the ethics committee of the affiliated hospital of Nanjing University of Chinese medicine.

\section{Operation Methods}

\section{Preoperative Preparations}

Improved preoperative preparations for general anesthesia. The development of nasal septum was evaluated by nasal CT, and the costal cartilage was reconstructed by three-dimensional CT to evaluate the calcification of costal cartilage. Trimmed nose hairs and cleaned nasal cavity before surgery. Had full communication with patients preoperatively in order to determine the operation plan. Septal cartilage and ear cartilage can be selected as repair materials to patients with good development of septal cartilage. For those with poor septal cartilage development and severe nasal dorsum collapse, costal cartilage is preferred as the repair material. If the costal cartilage calcification is obvious, avoid taking it.

Three quadrangular lengths were measured before surgery to determine the ideal nasal length, marked the nasal midline, the ideal radix point (the midpoint of the line between inner canthus and brows) and the ideal nasal tip defining point, designed the inverted "V" shape incision at the narrowest part of the columella and the alar cartilage rim incision, marked the costal cartilage or ear cartilage skin incision line. Three-dimensional images were taken with 3D camera before surgery, and the maximum distance from the long axis of the nostrils to the alar rim, the height of the nasal tip, the nasal tip-alar angle were measured. General anesthesia of conventional tracheal intubation, disinfected and drapped the surgical area. $0.5 \%$ lidocaine local anesthetic containing 1:200,000 epinephrine was prepared standby. 


\section{International Journal of Transplantation \& Plastic Surgery}

\section{Operation Technique}

\section{Cartilage taking and Carving}

The 7 th costal cartilage on the right side is usually selected, and the 6th costal cartilage is selected in a few cases. After local anesthesia was injected into the surgical area, skin and subcutaneous tissue were cut in turn according to the incision line, and a small amount of rectus abdominis fascia was excised standby. The muscle was separated obtusely, and the cartilage periosteum was cut and peeled off to expose the cartilage. If only alar and columellar strut graft were used, the entire costal cartilage with a length of about $3.0 \mathrm{~cm}$ was taken; if the grafts were also used for the nasal dorsal graft, the entire 7 th costal cartilage with a length of about $7.5 \mathrm{~cm}$ was taken. Strict hemostasis, drum lung experiment was taken to ensure there was no pleural injury. The muscle and subcutaneous tissue were sutured with circular needle and 4-0 silk thread, and the skin was sutured with 6-0 nylon thread. Costal cartilage and fascia were soaked in normal saline standby.

When taking the nasal septum cartilage, the nasal cavity was filled with gauze with epinephrine water to prevent blood flow and stop bleeding. Anesthesia was injected under the nasal mucosa on both sides of the nasal septum, and the nasal mucosa was cut from the right nostril $1.5 \mathrm{~cm}$ away from the front of the nasal septum and separated to the septal cartilage. The septal cartilage about $2.5 \mathrm{~cm} \times 1.5 \mathrm{~cm}$ in size was cut with a d-shaped knife. Care should be taken to protect the integrity of the nasal mucosa and to stop bleeding completely. The nasal septum cartilage was carved into pieces about $2.5 \mathrm{~cm} \times 0.4 \mathrm{~cm}$ in size and soaked in normal saline standby. When taking the ear cartilage, the posterior ear approach is generally selected to fully infiltrate and anesthetize the ventral and dorsal sides of the ear cartilage, so as to separate the skin and cartilage with water. The skin was cut $1.0 \mathrm{~cm}$ from the cranioauricular groove behind the ear, and the nail crest was retained to prevent auricle deformation. Cartilage about $2 \mathrm{~cm} \times 2.5 \mathrm{~cm}$ in size was cut from the cymba conchae and concha cavum respectively, and a small amount of posterior ear fascia was cut and soaked in normal saline standby.

\section{Correction of Nasal Soft-tissue Triangle Deformities}

After local anesthetic, cut the columella and the alar cartilage rim according to the incision line, separated the columella, nasal tip and nasal dorsum skin, the bilateral alar cartilage and lateral nasal cartilage were exposed, separated, released and dissociated the medial, middle and lateral crus of the alar cartilage, it could be seen that the alar cartilage at the dome was narrow and thin. The lateral rim retracted and collapsed. The supporting strength at the fornix was weak, and accompanied by dysplasia of the lateral crus. Measured and recorded the length and width of the alar cartilage and the angle between the medial and lateral crus.

Continued to separate and expose the lateral cartilages of the nose, separated a space between the nasal columella and the nasal dorsal fascia to the nasal spine and radix points. The sculpted costal cartilage or nasal septal cartilage can be vertically positioned in the columella space, straddling the tail of the septal cartilage, or overlapping with the tail by about $0.3 \mathrm{~cm}$, Used 5-0 PDS for u-suture. The other two pieces of costal cartilage or nasal septal cartilage were inserted longitudinally into both sides of the grafted cartilage of the nasal columella and the medial sides of the medial crus of the bilateral alar cartilage, and at the same time pulled and lifted the nasal cartilage on both sides. Needle fixation was used to estimate the position of the nasal tip, adjusted the nasal length and nasolabial angle to reach the ideal value, and sutured the graft cartilage and alar cartilage with 5-0 PDS to form a stable septal extension graft to strengthen the nasal columella support[8] (Figure 1b).

The costal cartilage slices or ear cartilage slices were transplanted above or below the middle and lateral crus of both sides of the alar cartilage, and the 6-0 PDS were used for interrupted suture and fixation to correct the retraction and collapse of the alar cartilage. By reducing the angle between the medial and lateral crus of the alar cartilage, the soft-tissue triangle was strengthened, and the shape of the alar dome was reconstructed, so as to extend the alar margin, improve the collapse of the softtissue triangle, adjusted the asymmetry of the soft-tissue triangle on both sides, and essentially correct the deformities of the soft-tissue triangle of the nose [1-3,5]. The radian of cartilage slices should be paid attention to during suture fixation, the angle of nasal tip should be controlled at about $60^{\circ}$, and the tension of both sides of the nasal alar should be balanced to avoid postoperative deviation (Figure 1c). After suturing, measured and recorded the angle of the medial and lateral crus of the alar cartilage.

Finally, according to the length and height of the nose, the rhinoplasty prosthesis or costal cartilage was carved into a willow leaf shape, with an appropriate length, width and thickness, and transplanted into the space below the dorsal fascia of the nose. The anterior end of the prosthesis was sutured and fixed with the alar 


\section{International Journal of Transplantation \& Plastic Surgery}

cartilage on both sides for several stitches, so as to correct the deformity of flat nose. Fascial tissue or untensioned ear cartilage was sutured and fixed in the appropriate position of the nasal tip to reduce the skin tension of the nasal tip, improved the tip defining point and the nasal column-lobular angle (Figure 1d). After checking the symmetry of bilateral nostrils and nasal alar, the 6-0 PDS was used for interrupted suture, and 1 negative pressure drainage was routinely placed in the wound cavity. Filled the nasal cavity with vaseline gauze and fixed it with thermoplastic plate on the nasal dorsum.

\section{Evaluation Indicators}

Comparison of the angle between the medial and lateral crus of the alar cartilage: The angle between the medial and lateral crus of the alar cartilage was carefully released and exposed during the operation. Real-time measurement of the angle was taken. After cartilage transplantation, nasal soft-tissue triangle support was strengthened by adjusting the angle between the medial and lateral crus of alar cartilage, and the angle was measured again when the reconstruction of alar cartilage dome was completed. This angle reflects the improvement of nasal alar collapse after surgery.

Comparison of the maximum distance from the long axis of the nostrils to the alar rim: The maximum distance from the long axis of the nostrils to the alar rim of the nose reflects the situation of alar retraction. Careful measurement of the distance and statistical analysis during preoperative and postoperative follow-up can help to estimate the degree of improvement of nasal alar retraction in soft-tissue triangle deformity.

Satisfaction survey on surgical efficacy: The appearance changes of the nose were observed by comparing the preoperative and postoperative photos. At the time of follow-up, a satisfaction questionnaire was issued, and a third party was invited to observe the patients, respectively evaluating the correction effect of nasal alar rim retraction, nasal soft-tissue triangle deformities, and bilateral asymmetry. According to different types of graft materials, it was divided into the pure costal cartilage group, the costal cartilage + prosthesis scaffold group, and the nasal septum cartilage + ear cartilage + prosthesis scaffold group. According to the patients' self-evaluation, doctors' judgment and thirdparty evaluation, the operation effect was comprehensively evaluated. If all three parties were satisfied, the curative effect should be judged as "significantly effective"; if two parties were satisfied, it should be deemed as "effective"; if one party was satisfied, it should be deemed as "generally effective"; if neither party was satisfied, it should be deemed as "poor"; if there were complications such as obvious infection and exposure of graft materials, it should be judged as "ineffective". Efficacy satisfaction = (number of significant effective cases + number of effective cases)/total number of cases $\times 100 \%$.

Statistical analysis: The angle between the medial and lateral crus of the alar cartilage and the maximum distance from the long axis of the nose to the alar edge

was expressed by $\mathrm{X} \pm$ s. One-way analysis of variance was used, and the statistical software was SPSS 16.0. $P<0.05$ was considered statistically significant.

\section{Result}

All these 32 patients with nasal soft-tissue triangle deformities, the angle between the medial and lateral crus of the alar cartilage was $(52.2 \pm 11.7)^{\circ}$ before surgery, and $(37.1 \pm 6.4)^{\circ}$ after the completion of reconstruction of the alar cartilage dome, the difference was statistically significant $(P<0.05)$. The maximum distance from the long axis of the nostrils to the alar rim was $(4.8 \pm 1.5) \mathrm{mm}$ before surgery and $(3.5 \pm 2.1) \mathrm{mm}$ after 6 months of follow-up, with statistically significant differences $(P<$ 0.05). The results showed that autologous cartilage grafts could repair cartilage collapse and/or retraction at the alar dome, extend the alar rim, and at the same time, repair the dome collapse by narrowing the angle between the medial and lateral crus, so as to achieve the goal of correcting nasal soft-tissue triangle deformities.

Patients were followed up for $6 \sim 18$ months, the noses were with natural appearance and tactile impression, including delicate tip defining point, the nasal dorsal and alar margins are gently transitioned, softtissue triangle deformity was significantly improved (see typical cases 1 and 2), Satisfaction on the correction effect of nasal soft-tissue triangle collapse was $87.5 \%$, alar rim retraction was $84.6 \%$, and asymmetry correction effect was 100\%. The overall postoperative effect was as follows: 21 cases were "significantly effective", 7 cases were "effective", and 3 cases were "generally effective". No "ineffective" cases were observed, no complications such as graft exposure, displacement, cartilage deformation, etc., and the overall efficacy satisfactory was as high as $87.5 \%$ (Table 1 ). 


\section{International Journal of Transplantation \& Plastic Surgery}

According to the different surgical graft materials, the efficacy was evaluated in different groups. The efficacy satisfaction was $100 \%$ in the pure costal cartilage group, $86.7 \%$ in the costal cartilage + prosthesis stent group, and $81.8 \%$ in the nasal septum cartilage + ear cartilage + prosthesis stent group. The efficacy satisfaction of the pure costal cartilage group was higher than that of the latter two groups, and the difference was statistically significant $(P<0.05)$. There was no statistically significant difference in satisfaction between the latter two groups $(P>0.05)$.

\begin{tabular}{|c|c|c|c|c|c|c|c|c|}
\hline \multirow{2}{*}{\multicolumn{2}{|c|}{ Classification }} & \multirow{2}{*}{$\begin{array}{l}\text { Number } \\
\text { of cases }\end{array}$} & \multicolumn{5}{|c|}{$\begin{array}{l}\text { Satisfaction evaluation (cases) } \\
\end{array}$} & \multirow{2}{*}{$\begin{array}{c}\text { Satisfactory } \\
(\%)\end{array}$} \\
\hline & & & $\begin{array}{l}\text { Significantly } \\
\text { effective }\end{array}$ & Effective & $\begin{array}{l}\text { Generally } \\
\text { effective }\end{array}$ & Poor & Ineffective & \\
\hline \multirow{3}{*}{$\begin{array}{c}\text { Surgical } \\
\text { site }\end{array}$} & $\begin{array}{l}\text { nasal soft-tissue triangle } \\
\text { collapse }\end{array}$ & 24 & 16 & 5 & 2 & 1 & 0 & 87.5 \\
\hline & alar rim retraction & 26 & 18 & 4 & 2 & 2 & 0 & 84.6 \\
\hline & asymmetry correction & 3 & 2 & 1 & 0 & 0 & 0 & 100 \\
\hline \multirow{4}{*}{$\begin{array}{c}\text { Graft } \\
\text { material }\end{array}$} & pure costal cartilage group & 6 & 5 & 1 & 0 & 0 & 0 & $100^{*}$ \\
\hline & $\begin{array}{c}\text { costal cartilage + prosthesis } \\
\text { stent group }\end{array}$ & 15 & 10 & 3 & 2 & 0 & 0 & 86.7 \\
\hline & $\begin{array}{c}\text { nasal septum cartilage + ear } \\
\text { cartilage + prosthesis stent } \\
\text { group }\end{array}$ & 11 & 6 & 3 & 1 & 1 & 0 & 81.8 \\
\hline & Overall efficacy & 32 & 21 & 7 & 3 & 1 & 0 & 87.5 \\
\hline
\end{tabular}

Table 1: Satisfaction score of patients after repairing nasal soft-tissue triangle deformities.

Note: Compared with the costal cartilage + prosthesis scaffold group and the septal cartilage + ear cartilage + prosthesis scaffold group, ${ }^{*} P<0.05$.

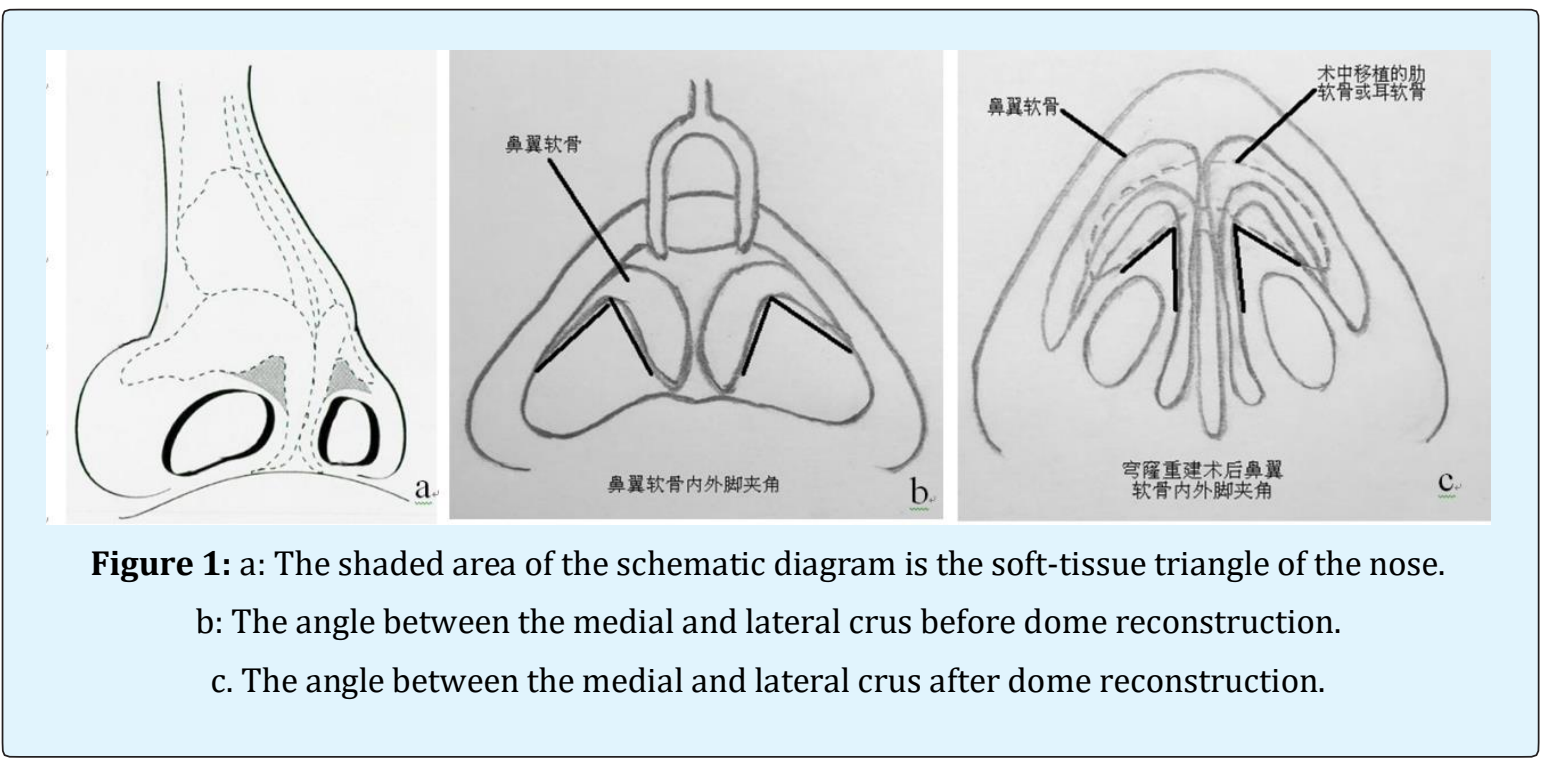

Huang J, et al. Application of Autologous Cartilage Transplantation in the Repair and Reconstruction of Nasal Soft-Tissue Triangle Deformities. Int J Transplant \& 


\section{International Journal of Transplantation \& Plastic Surgery}
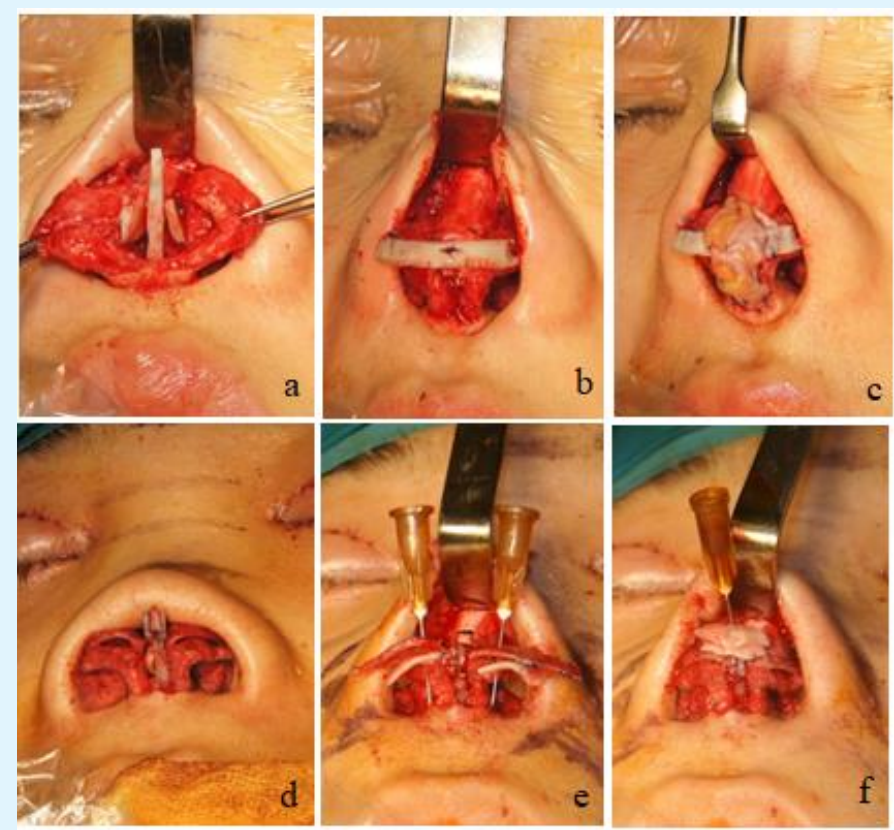

Figure 2: Schematic diagram of correction of nasal soft triangle deformities; a and b shows the correction method using autologous costal cartilage; c shows fascia tissue transplantation; $d, e, f$ is the correction method using autologous septal cartilage + ear cartilage.
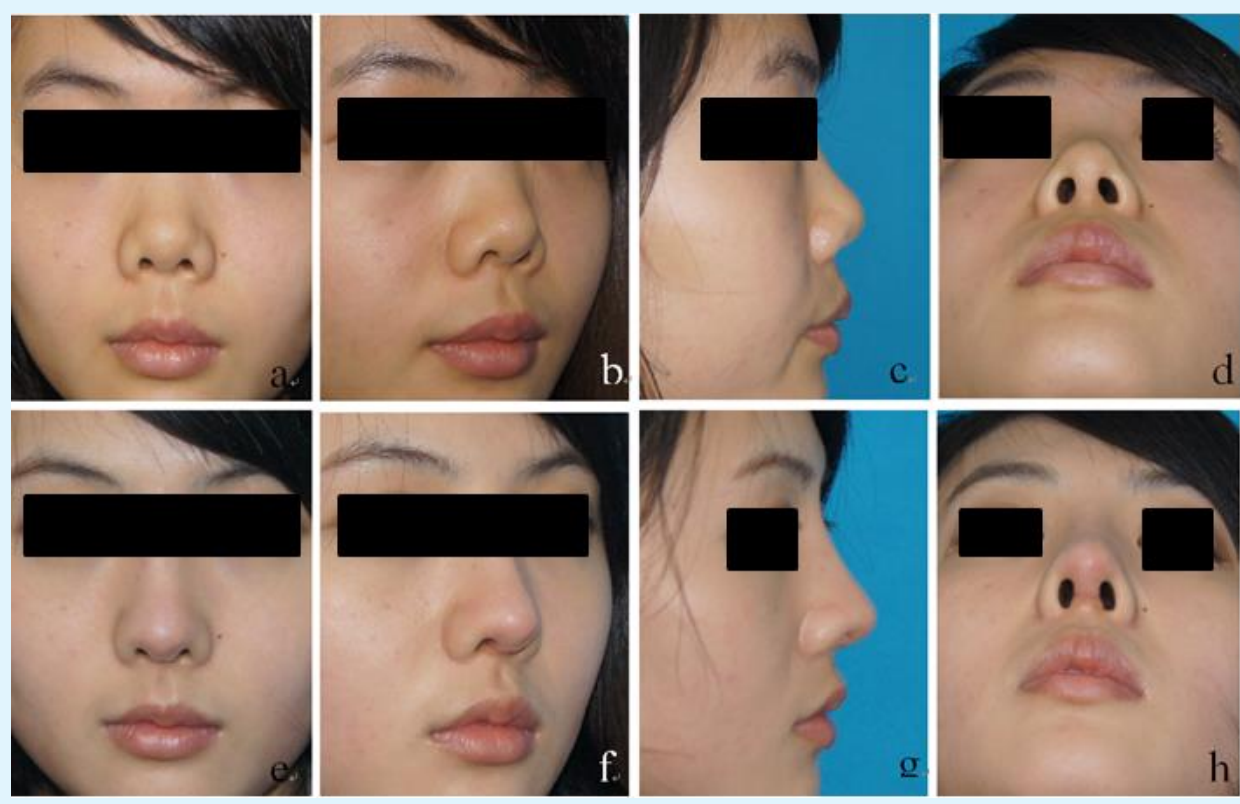

Figure 3: Typical case 1 : female, 21 years old, with soft-tissue triangle deformities combined with flat nose and short nose, the 7 th costal cartilage was taken intraoperatively, soft-tissue triangle deformities correction+shot nose deformity correction+e-PTFE augmentation rhinoplasty were carried out. (a, b, c, d for preoperative situation; e, f, g, h shows half a year after operative). 


\section{International Journal of Transplantation \& Plastic Surgery}
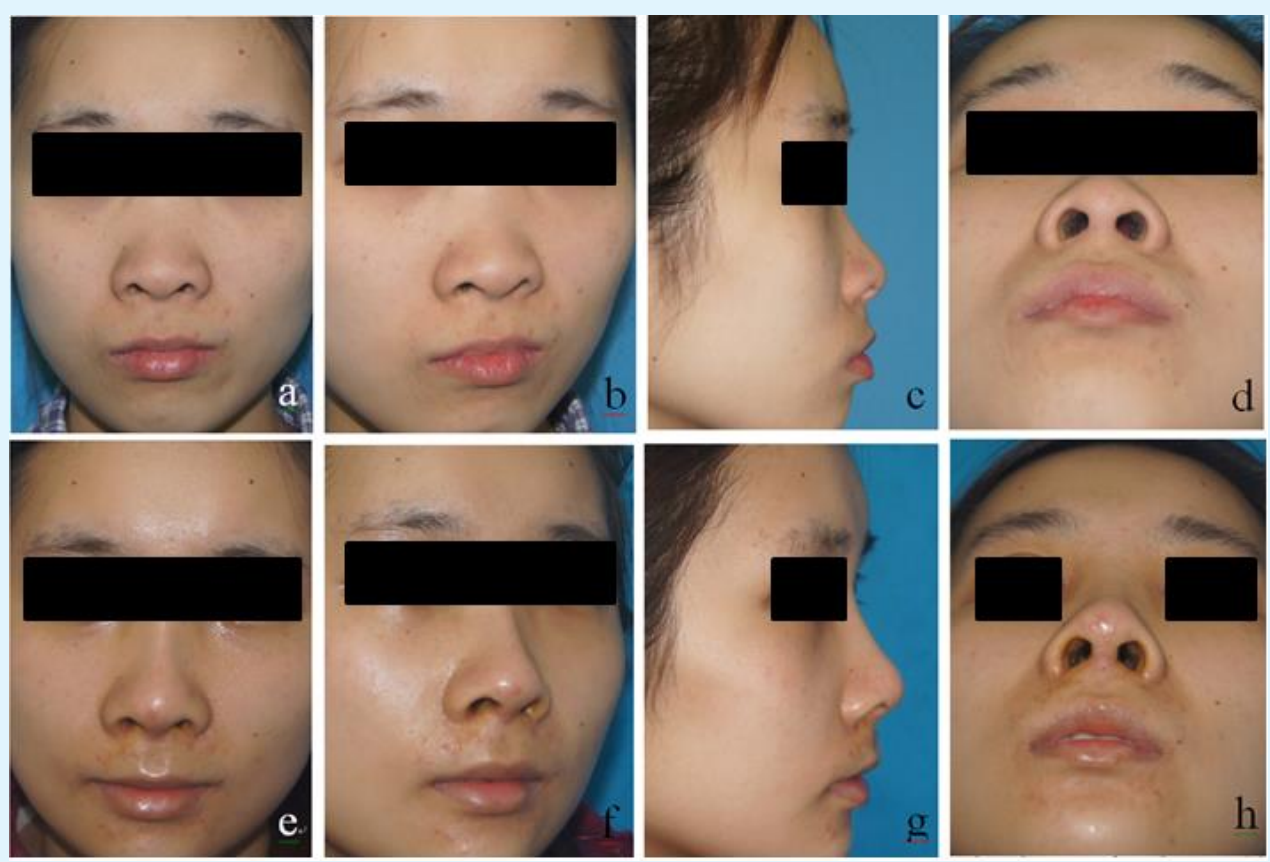

Figure 4: Typical case 2: female, 25 years old, with soft-tissue triangle deformities combined with flat nose deformity, nasal septal cartilage and ear cartilage were taken intraoperatively, soft-tissue triangle deformities correction+ ePTFE augmentation rhinoplasty were carried out (a, b, c, d for preoperative situation; e, f, g, h shows half a year after operative).

\section{Discussion}

The ideal external nasal morphology, the frontal view of the alar rim and nasal columella - lobular angle line, is similar to the gentle flying seagull wings. Lateral view shows oval nostril shap, the alar rim forms the upper portion of the ovoid $[9,10]$. Guyuron's study considered the normal relationship between the nasal alar and the nasal columella is that he maximum distance from the long nostril axis to the alar rim or the nasal columella rim was $1 \sim 2 \mathrm{~mm}$, while if the distance was larger than $2 \mathrm{~mm}$, it was characteristic nasal alar retraction [4]. Stable nasal tip and columellar support structures can partially counteract the retraction of the lateral crus, especially when the support structures have the effect of proloning the nose, it can push the lateral crus to the caudal side. Soft-tissue triangle deformities of the nose can be divided into congenitial deformities and secondary deformities, the clinical manifestations are soft-tissue triangle collapse, alar rim retraction or bilateral asymmetry, etc.

The pathological basis of congenital soft-tissue triangle deformities of the nose is determined by the anatomical characteristics of the region [4-6], while the pathological basis of secondary soft-tissue triangle deformities of the nose is caused by soft tissue or cartilage injury, defect and scar contracture caused by tumor, scald and trauma in the region [9-11]. All the cases in this study were patients with congenital soft triangle deformities of the nose. According to the above ideal external nasal morphology and data as the standard, whether there were problems such as alar retraction, alar collapse and/or bilateral asymmetry was determined. To correct these problems, a number of scholars have proposed cartilage transplantation to correct the cartilaginous aspects of nasal soft-tissue triangle deformities $[2,3,5]$. Due to the poor strength of the lateral crus in asians, even if good results are achieved under the action of stable tip and columellar support structure during the operation, the lateral crus may retract due to scar contracture and other problems during the recovery process. For secondary soft-tissue triangle deformities or defects of nose, local flap, adjacent flap or free transplantation of complex tissue are often used to do the repair [7,9-11]. 


\section{International Journal of Transplantation \& Plastic Surgery}

There is no rejection reaction after autologous cartilage transplantation, the tissue is easy to survive, less absorption, and is generally not easy to shift. The clinical sources of cartilage used in nasal cosmetology are mainly costal cartilage, nasal septum cartilage and ear cartilage. Among them, the costal cartilage or nasal septum cartilage is mainly used to reconstruct the nasal columella and nasal tip cartilage framework to form a stable nasal tip support complex, while ear cartilage is often used to correct the nasal tip. In patients with nasal soft-tissue triangle deformities, the defect and collapse of the alar cartilaginous dome are the main pathological basis, often accompanied by the collapse of the nasal tip, short nasal columella, flat nose, short nose and other problems [3]. When dealing with the soft-tissue triangle deformities of the nose, we need to correct other defects at the same time, which asks us to fully consider the cosmetic problems and treatment strategies of the nose into the design of surgery.

Based on the above theoretical basis, in this group, costal cartilage strips or ear cartilage were transplanted above the alar cartilage on both sides (Figure 1c) to repair the alar cartilage defect in the dome. At the same time, adjust the angle between the medial and lateral crus of the alar cartilage, reconstruct the shape of the dome of the alar cartilage, and strengthen the support of the lateral crus of the alar cartilage, so as to improve the retraction and collapse of the alar cartilage, and achieve the goal of correcting the soft-tissue triangle deformity of the nose. And at the same time improve the profile of the alar rim, showing the graceful curves of a seagull's gently flying wings.

Based on the above theoretical basis, in this group, costal cartilage strips or ear cartilage were transplanted above the alar cartilage on both sides (Figure 1c) to repair the alar cartilage defect in the dome. At the same time, adjust the angle between the medial and lateral crus of the alar cartilage, reconstruct the shape of the dome of the alar cartilage, and strengthen the support of the lateral crus of the alar cartilage, so as to improve the retraction and collapse of the alar cartilage, and achieve the goal of correcting the soft-tissue triangle deformity of the nose. And at the same time improve the profile of the alar rim, showing the graceful curves of a seagull's gently flying wings. Among them, there are many ways to adjust the angle medial and lateral cru of alar cartilage, such as the local flap of nasal column [10], the partial excision of alar cartilage [3], the suture of medial curus of alar cartilage to strengthen the support of nasal tip [8], and the transplantation of alar lateral crus cartilage, nasal septum cartilage and ear cartilage [2]. In this group, the latter two methods were mainly used. In addition, all cases in this group had problems with flat nose and/or short nose. Preoperative examination determined that nasal septal cartilage was not well developed. If nasal septal cartilage could not be taken to correct nasal deformities, autologous costal cartilage was designed to strengthen the support of nasal tip and nasal column and to augment the nasal tip, so as to correct short nose and lengthen nasal length.

In order to avoid the risk of nasal dorsal deformation and recollapse due to the absorption of costal cartilage, and to save the amount of cartilage available for transplantation, we chose e-PTFE or silicone prosthesis for augmentation rhinoplasty. According to the individual differences of the nose, customized sculpted prosthesis materials were transplanted into the subfascial space of the nasal dorsal to correct the nasal collapse deformity. Meanwhile, in order to cushion the excessive tension of nasal tip and nasal column support on the skin of the apex, appropriate volume of fascia tissue was transplanted on the nasal tip (Figure 2c). Long-term follow-up after the operation showed no complications such as redness, skin transparency and thinning of the nasal tip, or exposure of the graft materials.

Among the 23 patients with nasal soft-tissue triangle deformities in this group, there were 22 patients with alar rim retraction, 20 patients with nasal soft-tissue triangle collapse, and 2 patients with bilateral nasal soft-tissue triangle incomplete asymmetry. According to this, most patients had both nasal alar retraction and triangle collapse, and a few had asymmetric problems. For the evaluation of the postoperative efficacy of this group, the author referred to the evaluation method of Foda [2] and made some improvements. After intraoperative separation of alar cartilage, the angle of medial and laterial curs was firstly measured in the natural state. It could be seen that there were defects, retraction, collapse and asymmetry etc. of the cartilage in the tip of the anglethe alar cartilage in the dome. The suppot of the tip is weak, through the transplant of cartilage, first of all, it can mend the cartilage defects in front of the dome, in the process of cartilage suture fixation, by narrowing the angle between medial and laterial curs, to strengthen the support of dome cartilage, correct dome retraction, collapse and asymmetry problem, rebuild the shape of the alar cartilage dome. During the operation, the angle between medial and laterial curs of the alar cartilage was measured immediately, and the data is of high accuracy. 


\section{International Journal of Transplantation \& Plastic Surgery}

The change of the angle can objectively reflect the reconstruction of the alar dome. Statistical analysis of the data shows that there is a statistical difference in the change of the angle. At the follow-up, patients, doctors and a third party were asked to evaluate the alar rim retraction, nasal soft-tissue triangle deformities, bilateral asymmetry correction effect and overall surgical efficacy, and received high satisfactory.

There is still a poor evaluation of the efficacy in this research. Reasons may be as follows:

1. The size and shape of the grafted cartilage were not well designed and fixed, and the alar cartilage collapse and retraction correction were limited.

2. The nasal tip cartilaginous supporting complex has insufficient supporting power, which affects the shape of nasal tip.

3. The junction between the nasal soft-tissue triangle and the columella is not natural enough, and the alar rim curve is not that smooth and gentle.

In summary, autologous cartilage is suitable for the correction of nasal soft-tissue triangle deformities, and the combination of augmentation prosthesis and fascial tissue can effectively correct nasal collapse, short nose and other problems, with high postoperative satisfaction and low complications. This method has the value of clinical application and popularization.

\section{References}

1. Foda HM (2016) Nasal soft-tissue triangle deformities. Facial Plast Surg 32(4): 339-344.

2. Guyuron B, Ghavami A, Wishnek SM (2005) Components of the short nostril. Plast Reconstr Surg 116(5): 1517-1524.
3. Kayabasoglu G, Nacar A (2015) The soft triangle: An often neglected area in rhinoplasty. Aesthetic Plast Surg 39(5): 659-666.

4. Guyuron B (2003) Nasal tip sutures part II: the interplays]. Plast Reconstr Surg 112(4): 1130-1149.

5. Burget G (2013) Discussion: reconstruction of the nasal soft triangle subunit. Plast Reconstr Surg 131(5): 1051-1054.

6. Ali-Salaam P, Kashgarian M, Persing J (2002) The soft triangle revisited. Plast Reconstr Surg 110(1): 14-16.

7. Constantine FC, Lee MR, Sinno S (2013) Reconstruction of the nasal soft triangle subunit. Plast Reconstr Surg 131(5): 1045-1050.

8. Huang J, Liu Y (2012) A modified technique of septal extension using a septal cartilage graft for short-nose rhinoplasty in Asians. Aesthetic Plast Surg 36(5): 1028-1038.

9. Li Y, Sepulveda A, Buchanan EP (2015) Late presenting nasal deformities after nasal continuous positive airway pressure injury: 33-year experience. J Plast Reconstr Aesthet Surg 68(3): 339-343.

10. Ersoy B, Celebiler O, Numanoğlu A (2010) A new alternative for reconstruction of soft triangle defects secondary to burn injury: superiorly based columellar flap. J Plast Reconstr Aesthet Surg 63(10): 1733-1735.

11. Moloney GR, Rutherford T (2013) Cheek interpolation flap and composite graft repair of a nasal tip and fullthickness soft triangle defect. Dermatol Surg 39(9): 1390-1393. 